TRANSACTIONS OF THE

AMERICAN MATHEMATICAL SOCIETY

Volume 349, Number 4, April 1997, Pages 1663-1674

S 0002-9947(97)01641-3

\title{
ONE AND TWO DIMENSIONAL CANTOR-LEBESGUE TYPE THEOREMS
}

\author{
J. MARSHALL ASH AND GANG WANG
}

\begin{abstract}
Let $\varphi(n)$ be any function which grows more slowly than exponentially in $n$, i.e., limsup $\varphi(n)^{1 / n} \leq 1$. There is a double trigonometric series whose coefficients grow like $\varphi(n)$, and which is everywhere convergent in the square, restricted rectangular, and one-way iterative senses. Given any preassigned rate, there is a one dimensional trigonometric series whose coefficients grow at that rate, but which has an everywhere convergent partial sum subsequence. There is a one dimensional trigonometric series whose coefficients grow like $\varphi(n)$, and which has the everywhere convergent partial sum subsequence $S_{2^{j}}$. For any $p>1$, there is a one dimensional trigonometric series whose coefficients grow like $\varphi\left(n^{\frac{p-1}{p}}\right)$, and which has the everywhere convergent partial sum subsequence $S_{\left[j^{p}\right]}$. All these examples exhibit, in a sense, the worst possible behavior. If $m_{j}$ is increasing and has arbitrarily large gaps, there is a one dimensional trigonometric series with unbounded coefficients which has the everywhere convergent partial sum subsequence $S_{m_{j}}$.
\end{abstract}

\section{INTRODUCTION}

The original Cantor-Lebesgue theorem was a statement about sequences: If $\left\{A_{n}(x)\right\}:=\left\{c_{n} e^{i n x}+c_{-n} e^{-i n x}\right\}$ tends to zero at each point of a set of positive one-dimensional measure as $n \rightarrow \infty$, then $\left\{\left|c_{n}\right|+\left|c_{-n}\right|\right\}$ tends to 0 as $n \rightarrow \infty$. (Here and henceforth, measure will mean Lebesgue measure.)

A frequently used corollary, whose statement will be the starting point of our investigations, is the following.

Theorem 1 (Cantor-Lebesgue). If a trigonometric series $c_{o}+\sum_{n=1}^{\infty} A_{n}(x)$ converges at each point $x$ of a set of positive one-dimensional measure as $n \rightarrow \infty$, then $\left\{\left|c_{n}\right|+\left|c_{-n}\right|\right\}$ tends to 0 as $n \rightarrow \infty$.

Proof. If a series converges, then its $n$-th term tends to 0 . Now apply the original Cantor-Lebesgue theorem.

Received by the editors February 23, 1994 and, in revised form, November 20, 1995.

1991 Mathematics Subject Classification. Primary 42A20, 42B99; Secondary 40A05, 40C99.

Key words and phrases. Cantor-Lebesgue theorem, coefficient size, subsequences, trigonometric series, two dimensional trigonometric series, restricted rectangular convergence.

J. M. Ash was partially supported by the National Science Foundation grant no. DMS-9307242. G. Wang was partially supported by grants from the Faculty Research and Development Program of the College of Liberal Arts and Sciences, DePaul University.

(C) 1997 American Mathematical Society 
Probably the first, and still one of the best, applications of Theorem 1 was Cantor's uniqueness theorem. Cantor wished to prove that if a trigonometric series converges to 0 everywhere, then it must be the trivial series. (All $c_{n}=0$.) Essentially his proof consisted of two steps.

Step 1. If a trigonometric series converges everywhere, then $\left\{\left|c_{n}\right|+\left|c_{-n}\right|\right\}$ tends to 0 as $n \rightarrow \infty$.

Step 2. If a trigonometric series converges to 0 everywhere, and if its coefficients satisfy the condition that

$$
\left\{\left|c_{n}\right|+\left|c_{-n}\right|\right\} \text { tends to } 0 \text { as } n \rightarrow \infty,
$$

then it must be the trivial series.

Note that Step 1 is a weak version of Theorem 1. The main reason for our interest in seeing how far Theorem 1 can be extended into multiple trigonometric series is that all known uniqueness proofs (and there are several, since both dimension and mode of convergence can vary) either pass through the above two steps, or else just assume a growth condition similar to condition (1).

To illustrate this, in dimension $d, d>1$, take the mode of convergence to be spherical. Here there is a very nice analogue of Theorem 1 . In fact, it follows from work of Cooke (when $d=2$ ) [C3] and Connes (when $d>2$ ) [C2] that if at each $x$ in $T^{d}:=(-\pi, \pi]^{d}$ we have

$$
\lim _{R \rightarrow \infty} \sum_{|m|=R} c_{m} e^{i m x}=0,
$$

where $|m|:=\sqrt{\sum m_{i}^{2}}$, then

$$
\lim _{R \rightarrow \infty} \sum_{|m|=R}\left|c_{m}\right|^{2}=0
$$

In particular, the coefficients $c_{m}$ tend to 0 as $|m| \rightarrow \infty$. Indeed, the analogue of Step 2 is also valid. This was shown by Shapiro (when $d=2$ ) [S] and (very recently) Bourgain (when $d>2$ ) [B].

If $m=\left(m_{1}, \ldots, m_{d}\right), n=\left(n_{1}, \ldots, n_{d}\right)$ and $m_{i} \geq n_{i}$ for all $i$, write $m \geq n$. Let $0=(0, \ldots, 0)$ and define

$$
A_{n}(x):=\sum_{\left\{\left|m_{i}\right|=n_{i}, i=1, \ldots, d\right\}} c_{m} e^{i m \cdot x}
$$

so that the sum has $2^{s}$ terms, where $s$ is the number of non-zero components of $n$. Also define

$$
\rho_{n}:=\sum_{\left\{\left|m_{i}\right|=n_{i}, i=1, \ldots, d\right\}}\left|c_{m}\right|,
$$

$\|m\|:=\min \left\{\left|m_{i}\right|: i=1, \ldots, d\right\}$, and $\|m\|:=\max \left\{\left|m_{i}\right|: i=1, \ldots, d\right\}$. Notice that if $\|m\|$ is large, $m$ is neither near the origin nor near any coordinate axis, while $\|m\|=$ $k$ only means that $m$ is on the edge of an origin-centered $\underbrace{2 k \times \ldots \times 2 k}_{d \text { terms }}$ cube. Three methods of convergence of multiple trigonometric series will now be considered. A multiple trigonometric series is square convergent at $x$ if $\lim _{N \rightarrow \infty} \sum_{\|n\| \leq N} A_{n}(x)$ exists. It is unrestrictedly rectangularly convergent at $x$ if the rectangular partial sums 
$T_{n}(x):=\sum_{0 \leq m \leq n} A_{m}(x)$ are such that $\lim _{\|n\| \rightarrow \infty} T_{n}(x)$ exists; and it is restrictedly rectangularly convergent at $x$ if there is a complex number $s$ such that for each (arbitrarily large) $E>1$ the limit of $T_{n}(x)$ is $s$ as $\|n\| \rightarrow \infty$ in such a way that every ratio $n_{i} / n_{j}$ is bounded by $E$.

The following results are known:

Theorem 2. If $T(x)=\sum_{n \geq 0} A_{n}(x)$ converges unrestrictedly rectangularly for all $x$ in a positive measured subset of $T^{d}$, then (1) the $\left\{c_{m}\right\}$ in equation (2) are bounded, and (2) $\left\{\rho_{n}\right\}$ tends to 0 as $\|n\| \rightarrow \infty[\mathrm{AW}, 410]$.

Theorem 3. If $T(x)=\sum_{n \geq 0} A_{n}(x)$ is square convergent for all $x$ in a full measured subset of $T^{d}$, and if $\gamma>1$ is given, then there exists $b=b(T, \gamma)>0$ such that $\left|c_{m}\right| \leq b \gamma\|m\|[\mathrm{AW}, 408]$.

Theorem 4. If $T(x)=\sum_{n \geq 0} A_{n}(x)$ converges restrictedly rectangularly for all $x$ in a full measured subset of $T^{d}$, and if $\gamma>1$ is given, then (1) there exists $b=b(T, \gamma)>0$ such that $\left|c_{m}\right| \leq b \gamma\|m\|$, and (2) given any $E>1,\left\{\rho_{n}\right\}$ tends to 0 as $\|n\| \rightarrow \infty$ in such a way that every ratio $n_{i} / n_{j}$ is bounded by $E$.

Theorem 2's conclusion (1) is nontrivial when $d \geq 2$ : consider the unrestrictedly rectangularly convergent numerical series $s=\sum_{m, n=0}^{\infty} a_{m n}$ where $a_{0 n}=2^{n}, a_{1 n}=$ $-2^{n}, a_{m n}=0$ otherwise. The conclusions of this theorem are strong enough to allow the verification of the analogue of Step 2 when the mode of convergence is unrestricted rectangular [AFR].

Theorem 3 was proved by Paul Cohen [C1]. We reference [AW] above since Cohen's (very good and very interesting) thesis, although available in the University of Chicago Library, was never published.

Theorem 4 is not explicitly written down anywhere, but conclusion (1) is a consequence of the previous theorem since restricted rectangular convergence at a point implies square convergence there, and conclusion (2) is proved by "Mondrianing" in a way that is quite similar to the proof of conclusion (2) of Theorem 2. (See pages $410-411$ of $[\mathrm{AW}]$.)

Notice that the conclusions of Theorems 3 and 4 are both quite weak and consequently of little use in establishing uniqueness theorems without coefficient growth hypotheses. The motivation for this paper is to give an example of a double trigonometric series which converges everywhere to an everywhere finite-valued function in the sense of restricted rectangular convergence (and, consequently, also in the sense of square convergence), but whose coefficient growth is so bad as to demonstrate that the seemingly puny conclusions of Theorems 3 and 4 would remain best possible, even if their hypotheses were strengthened by removing the modifying phrase "a full measured subset of".

More precisely, say that $\varphi$ grows more slowly than exponentially in $n$ if for any given $\gamma>1$ there exists $b=b(\varphi, \gamma)>0$ such that $|\varphi(n)| \leq b \gamma^{n}$. Note that $\varphi$ grows more slowly than exponentially in $n$ if and only if $\limsup _{n \rightarrow \infty} \varphi(n)^{1 / n} \leq 1$.

For example, $\varphi(n)$ could be $n^{1000000}$ or $e^{n / \log \log n}$. By grow like $\varphi(n)$, we mean that there is a constant $C$ and a subsequence of the coefficients $\left\{c_{n_{i}}\right\}$ satisfying $C^{-1} \varphi\left(\left\|n_{i}\right\|\right) \leq\left|c_{n_{i}}\right| \leq C \varphi\left(\left\|n_{i}\right\|\right)$. Theorem 5 of Section 2 asserts that given any 
function which grows more slowly than exponentially, there is a double trigonometric series whose coefficients grow like it, and which is everywhere restrictedly rectangularly convergent.

Passing to one dimension, in Section 3 we ask to what extent the CantorLebesgue theorem holds for subsequences. In general, no positive result is possible. More explicitly, given any natural-number-domained function $\psi(n)$, Theorem 6 produces a one-dimensional trigonometric series of power series type whose coefficients grow like $\psi(n)$. Nevertheless, this series has an everywhere convergent subsequence.

However, for certain particular subsequences, there are some best possible controls on the allowable rate of growth. For example, if $m_{j}=2^{j}$, and if we are given a function $\varphi(n)$ growing more slowly than exponentially, then Theorem 7 says that there is a trigonometric series which has the subsequence of $2^{j}$-th partial sums converging everywhere, but whose coefficients grow like $\varphi(n)$. This result is best possible in the sense that if the $2^{j}$-th partial sums of any trigonometric series converge almost everywhere, then the coefficients of that series must grow more slowly than exponentially. Similarly, if $m_{j}=\left[j^{p}\right]$, where $p>1$, then Theorem 9 says that the maximum possible rate of growth for the coefficients of a trigonometric series which has the subsequence of $m_{j}$-th partial sums converging everywhere is exactly

$\varphi\left(n^{\frac{p-1}{p}}\right)$, where $\varphi(n)$ grows more slowly than exponentially. Many results for other special subsequences can be proved similarly.

The counterexample technique used in the last paragraph also leads in Theorem 10 to the fact that for any sequence $\left\{n_{k}\right\}$ containing arbitrarily large gaps, there is a trigonometric series whose $n_{k}$-th partial sums converge everywhere, but whose coefficients are unbounded. (Compare example 5 of $[\mathrm{AKR}]$.)

\section{The TWO DIMENSIONAL EXAMPLE}

Theorem 5. Let $\varphi(n)$ be any function which grows more slowly than exponentially in $n$. Then there is a double trigonometric series whose coefficients grow like $\varphi(n)$, and which is everywhere convergent to an everywhere finite function in the square, restricted rectangular, and one-way iterative senses.

Proof. We will construct an example of the form $\sum_{n=4}^{\infty} \sum_{m=0}^{\infty} t_{m n} \cos m x \cos n y$ and show that the appropriate convergence occurs and that $t_{0 n} \simeq \varphi(n)$. Of course, if we were to rewrite the series in the form $\sum_{|n| \geq 4} \sum_{m=-\infty}^{\infty} c_{m n} e^{i(m x+n y)}$, the convergence properties would persist and we would have $c_{0 n}=c_{0-n}=t_{0 n} / 2 \simeq \varphi(n) / 2$.

The example series is

$$
T(x, y):=2 \sqrt{\pi} \sum_{n=2}^{\infty} n^{3 / 2} \varphi(n) \cos ^{2}\left(\frac{x}{2}\right) \sin ^{2 n-2}\left(\frac{x}{2}\right) \cos n y .
$$

Let $T_{N}(x, y):=2 \sqrt{\pi} \sum_{n=1}^{N} n^{3 / 2} \varphi(n) \cos ^{2}\left(\frac{x}{2}\right) \sin ^{2 n-2}\left(\frac{x}{2}\right) \cos n y$ be the partial sum of this (one-dimensional) series. Let $S_{\varepsilon}:=(-\pi+\varepsilon, \pi-\varepsilon) \times(-\pi, \pi]$. We first establish the following claim.

For each small $\varepsilon>0, T_{N}$ converges uniformly to a continuous function on $S_{\varepsilon}$. 
Letting $\gamma(\varepsilon):=\sin ^{2}\left(\frac{\pi-\varepsilon}{2}\right)$ and estimating $\left|\cos ^{2}\left(\frac{x}{2}\right) \cos n y\right|$ by 1 we see that for $(x, y) \in S_{\varepsilon}$, the $n$-th term of $T(x, y)$ is dominated by $2 \sqrt{\pi} n^{3 / 2} \varphi(n) \gamma(\varepsilon)^{n-1}$. By the root test, $\sum_{n=1}^{\infty} n^{3 / 2} \varphi(n) \gamma(\varepsilon)^{n-1}$ converges, since $\limsup _{n \rightarrow \infty}\left(n^{3 / 2} \varphi(n) \gamma(\varepsilon)^{n-1}\right)^{1 / n}=$ $\gamma(\varepsilon)<1$. Apply the Weierstrass $M$-test to conclude that statement (3) holds.

It follows immediately from statement (3) that $T_{N}(x, y)$ converges on $T^{2} \backslash\{(\pi, y)$ : $-\pi<y \leq \pi\}$. However, on the exceptional line, we have $x=\pi$ and $\cos ^{2}(\pi / 2)=0$, so that $T_{N}$ converges (to 0 ) there also. We will pedantically distinguish between the function $\hat{T}(x, y):=\lim _{N \rightarrow \infty} T_{N}(x, y)$ and the formal series $T(x, y)$.

The trigonometric identities

$$
\begin{aligned}
\sin ^{2 n-2}\left(\frac{x}{2}\right) & =(2 i)^{-2 n+2} \sum_{k=0}^{2 n-2}\left(\begin{array}{c}
2 n-2 \\
k
\end{array}\right) e^{i(2 n-2-k) x / 2} e^{-i k x / 2}(-1)^{k} \\
& =2^{-2 n+2}\left\{\left(\begin{array}{c}
2 n-2 \\
n-1
\end{array}\right)+2 \sum_{m=1}^{n-1}\left(\begin{array}{c}
2 n-2 \\
n-1-m
\end{array}\right)(-1)^{m} \cos m x\right\}
\end{aligned}
$$

and

lead to

$$
4 \cos ^{2}\left(\frac{x}{2}\right) \cos m x=\cos (m+1) x+2 \cos m x+\cos (m-1) x
$$

$$
\begin{aligned}
4^{n} \cos ^{2} & \left(\frac{x}{2}\right) \sin ^{2 n-2}\left(\frac{x}{2}\right) \\
= & \left(\begin{array}{c}
2 n \\
n
\end{array}\right) \frac{1}{(2 n-1)}+\left(\begin{array}{c}
2 n \\
n+1
\end{array}\right) \frac{(n+4)(n-1)}{n(2 n-1)}(-1) \cos x \\
& +2 \sum_{m=2}^{n-2}\left(\begin{array}{c}
2 n \\
n+m
\end{array}\right) \frac{n-2 m^{2}}{n(2 n-1)}(-1)^{m} \cos m x \\
& +\left(\begin{array}{c}
2 n \\
2 n-1
\end{array}\right) \frac{4-2 n}{n}(-1)^{n-1} \cos (n-1) x-2\left(\begin{array}{c}
2 n \\
2 n
\end{array}\right)(-1)^{n} \cos n x
\end{aligned}
$$

For $n \geq 4$, we set

$$
\begin{aligned}
b_{0, n} & :=\frac{1}{2 n-1}, \\
b_{1, n} & :=\frac{(n+4)(n-1)}{n(2 n-1)}, \\
b_{m n} & :=2 \frac{n-2 m^{2}}{n(2 n-1)} \text { for } 2 \leq m \leq n-2, \\
b_{n-1, n} & :=\frac{4-2 n}{n}, \\
b_{n n} & :=-2, \\
b_{m n} & :=0 \text { for all other }(m, n),
\end{aligned}
$$

and

$$
t_{m n}:=2 \sqrt{\pi} n^{3 / 2} \varphi(n) 4^{-n}\left(\begin{array}{c}
2 n \\
n+m
\end{array}\right)(-1)^{m} b_{m n} .
$$

Since $t_{m n}=0$ when $(m, n)$ is not in the triangular region $\{(m, n): 0 \leq m \leq$ $n\}, T$ may be written in the form $\sum_{n=4}^{\infty} \sum_{m=0}^{n} t_{m n} \cos m x \cos n y$. Thus $T$ may be thought of as a double trigonometric series with rectangular partial sums $T_{M N}:=$ 
$\sum_{n=4}^{N} \sum_{m=0}^{M} t_{m n} \cos m x \cos n y$ and $T_{N}$ may be thought of as $T_{N N}$, the square partial sum of $T$. Thus $T$ is square convergent everywhere. Also

$$
T_{N}=\sum_{n=4}^{N}\left\{\sum_{m=0}^{\infty} t_{m n} \cos m x\right\} \cos n y
$$

where the inner sum is only formally infinite and so may be thought of as the $(\infty, N)$ iterated partial sum of $T$. In other words, $T$ is one-way iteratedly convergent everywhere in the sense that

$$
\hat{T}(x, y)=\lim _{N \rightarrow \infty} \sum_{n=4}^{N}\left\{\lim _{M \rightarrow \infty} \sum_{m=0}^{M} t_{m n} \cos m x\right\} \cos n y
$$

exists as a finite real number for each $(x, y)$.

Using Stirling's formula, $\Gamma(x+1) \simeq \sqrt{2 \pi x} x^{x} e^{-x}$ as $x$ tends to $+\infty$ through real values, we find that

$$
t_{0, n}:=2 \sqrt{\pi} n^{3 / 2} \varphi(n) \frac{\left(\begin{array}{c}
2 n \\
n
\end{array}\right) \frac{1}{2 n-1}}{2^{2 n}}=\sqrt{\pi} \frac{2 n^{3 / 2}}{2 n-1} \frac{\varphi(n)}{2^{2 n}} \frac{\Gamma(2 n+1)}{\Gamma(n+1)^{2}} \simeq \varphi(n),
$$

which shows that the coefficients indeed grow like $\varphi(n)$.

It only remains to show that $T$ converges restrictedly rectangularly to $\hat{T}$. First note that if $M \geq N$, then $T_{M N}=T_{N N}=T_{N}$, i.e., short, fat partial sums of $T$ are really just square partial sums. Consequently, for all $(x, y)$,

$$
\lim _{M, N \rightarrow \infty, M \geq N} T_{M N}(x, y)=\hat{T}(x, y) .
$$

Fix (a very large) $E>1$. We must show that

$$
\lim _{M \rightarrow \infty, E \geq N / M>1} T_{M N}(x, y)=\hat{T}(x, y) .
$$

Consider a partial sum $T_{M N}(x, y)$ with $M E \geq N>M \geq 1$. Because of the triangular nature of $\left\{(m, n): t_{m n} \neq 0\right\}$

$$
T_{M N}=T_{N N}-\sum_{n=M+1}^{N} \sum_{m=M+1}^{n} t_{m n} \cos m x \cos n y .
$$

Since for each $(x, y), T_{N N} \rightarrow \hat{T}$, to establish equation (4), it suffices to prove that

$$
\lim _{M \rightarrow \infty, E \geq N / M>1} \sum_{n=M+1}^{N} \sum_{m=M+1}^{n}\left|t_{m n}\right|=0 .
$$

Now

$$
\begin{aligned}
& \sum_{m=M+1}^{n}\left|t_{m n}\right| \\
& \quad=2 \sqrt{\pi} n^{3 / 2} \varphi(n) 4^{-n}\left(2 \sum_{m=M+1}^{n-2}\left(\begin{array}{c}
2 n \\
n+m
\end{array}\right)\left|\frac{n-2 m^{2}}{n(2 n-1)}\right|+4(n-2)+2\right) .
\end{aligned}
$$

We may estimate this quite roughly. The binomial coefficients are decreasing as $m$ increases, so estimating the quantity in absolute values by 1 , and then replacing 
every term in the sum by the largest one, and finally estimating the number of terms in the sum by $n$, we arrive at

$$
\sum_{m=M+1}^{n}\left|t_{m n}\right| \leq C \frac{n^{5 / 2} \varphi(n)}{4^{n}}\left(\begin{array}{c}
2 n \\
n+M+1
\end{array}\right)
$$

so that the left side of relation (5) is bounded by

$$
\lim _{M \rightarrow \infty, E \geq N / M>1} \sum_{n=M+1}^{N} C \frac{n^{5 / 2} \varphi(n)}{4^{n}}\left(\begin{array}{c}
2 n \\
n+M+1
\end{array}\right) .
$$

Note that $n+M+1>n+N / E>n(1+1 / E)=: n \rho$, where $2>\rho>1$. For $x \in[0, n]$ real, define

$$
\left(\begin{array}{l}
n \\
x
\end{array}\right):=\frac{n !}{\Gamma(x+1) \Gamma(n-x+1)} .
$$

Since, as was pointed out to us by Richard Askey, the quantity

$$
\frac{\partial}{\partial x} \log \left(\begin{array}{l}
n \\
x
\end{array}\right)=\frac{\Gamma^{\prime}}{\Gamma}(n-x+1)-\frac{\Gamma^{\prime}}{\Gamma}(x+1)=\left\{\frac{n}{2}-x\right\} \sum_{k=0}^{\infty} \frac{2}{(k+x+1)(k+n-x+1)}
$$

is negative for $x \in\left(\frac{n}{2}, n\right)$, it follows that $\left(\begin{array}{c}2 n \\ \rho n\end{array}\right)>\left(\begin{array}{c}2 n \\ n+M+1\end{array}\right)$. Then relation (5) will hold provided

$$
\lim _{M \rightarrow \infty} \sum_{n=M+1}^{\infty} \frac{n^{5 / 2} \varphi(n)}{4^{n}}\left(\begin{array}{c}
2 n \\
\rho n
\end{array}\right)=0 .
$$

But

$$
\begin{aligned}
\frac{1}{4^{n}}\left(\begin{array}{c}
2 n \\
\rho n
\end{array}\right) & =\frac{(2 n) !}{4^{n} \Gamma(\rho n+1) \Gamma((2-\rho) n+1)} \\
& \simeq \frac{1}{\sqrt{2 \pi n}} \frac{1}{\rho^{1 / 2}(2-\rho)^{1 / 2}}\left(\frac{1}{\rho^{\rho}(2-\rho)^{2-\rho}}\right)^{n} \\
& \leq \frac{C_{\rho}}{n^{1 / 2}}\left(\gamma_{\rho}\right)^{n},
\end{aligned}
$$

where $\gamma_{\rho}:=\rho^{-\rho}(2-\rho)^{\rho-2}$. The computation $\left(\log \gamma_{r}\right)^{\prime}=\log \left(\frac{2}{r}-1\right)<0$ for $r \in(1,2)$ implies that $\gamma_{\rho}<\gamma_{1}=1$. Thus the sum on the left side of relation (6) is bounded by $\sum_{n=M+1}^{\infty} C_{\rho} n^{2} \varphi(n)\left(\gamma_{\rho}\right)^{n}$. Since $\limsup _{n \rightarrow \infty}\left(C_{\rho} n^{2} \varphi(n)\left(\gamma_{\rho}\right)^{n}\right)^{1 / n} \leq \gamma_{\rho}<1$, by the root test this last sum is the tail of a convergent series and thus tends to 0 as $M \rightarrow \infty$. Theorem 5 is proved.

\section{One Dimensional EXAMPLES AND THEOREMS}

Theorem 6. Given any positive sequence $\psi(n)$, there is an increasing sequence of integers $\left\{m_{j}\right\}$ and a trigonometric series of power series type $S=\sum_{n=0}^{\infty} c_{n} e^{i n x}$ such that the $\left(m_{j}-1\right)$ th partial sums of $S$ are everywhere convergent, but for every $j$, $c_{m_{j}}=\psi\left(m_{j}\right)$.

Proof. Identify the torus with the edge of the unit circle in the complex plane via the mapping $x \rightarrow z=e^{i x}$. For $j \geq 1$, decompose the torus into $F_{j}=\left\{e^{i x}: 0<x<1 / j\right\}$ and its complement $K_{j}$. Let $m_{1}=1$. The function $1 / z$ is continuous on $K_{1}$, the set $K_{1}$ is compact and has no interior, and the complement of $K_{1}=\{z:|z| \neq 1\} \cup F_{1}$ 
is connected, so by a theorem of Mergelyan ([R], page 390), there is a trigonometric polynomial $\sum_{k=0}^{n_{1}} d_{k} e^{i k x}$ satisfying

$$
\sup _{x \in K_{1}}\left|e^{-i x}-\sum_{k=0}^{n_{1}} d_{k} e^{i k x}\right|<\frac{1}{\psi\left(m_{1}\right) 2} .
$$

Multiplying by $e^{i x}$ gives

$$
\sup _{x \in K_{1}}\left|1-\sum_{k=1}^{n_{1}+1} d_{k-1} e^{i k x}\right|<\frac{1}{\psi\left(m_{1}\right) 2} .
$$

Let $Q_{1}(x)=1-\sum_{k=1}^{n_{1}+1} d_{k-1} e^{i k x}$. Now let $m_{2}=n_{1}+3=m_{1}+\operatorname{deg} Q_{1}+1$. By Mergelyan's theorem again, there is a trigonometric polynomial $Q_{2}(x)$ with constant term equal to 1 such that

$$
\sup _{x \in K_{2}}\left|Q_{2}(x)\right|<\frac{1}{\psi\left(m_{2}\right) 2^{2}} .
$$

Proceeding inductively, having chosen a trigonometric polynomial $Q_{j}(x)$ with constant term equal to 1 such that

$$
\sup _{x \in K_{j}}\left|Q_{j}(x)\right|<\frac{1}{\psi\left(m_{j}\right) 2^{j}},
$$

define $m_{j+1}=m_{j}+\operatorname{deg} Q_{j}+1$ and then use Mergelyan's theorem to approximate $e^{-i x}$ on $K_{j+1}$ to within $\frac{1}{\psi\left(m_{j+1} 2^{j+1}\right.}$. Then a multiplication by $e^{i x}$ produces $Q_{j+1}$, a trigonometric polynomial with constant term equal to 1 satisfying

$$
\sup _{x \in K_{j+1}}\left|Q_{j+1}(x)\right|<\frac{1}{\psi\left(m_{j+1}\right) 2^{j+1}} .
$$

The required counterexample is

$$
S=\sum_{j=1}^{\infty} \psi\left(m_{j}\right) e^{i m_{j} x} Q_{j}(x) .
$$

The $m_{j}$ were chosen so that the last frequency appearing in the $j$ th term of this series is exactly $m_{j+1}-1$, which is 1 less than the first frequency of the next term of this series. Thus the $k$ th partial sum of $S$ as written in formula (7) is the $\left(m_{k+1}-1\right)$ th partial sum of $S$ thought of as a trigonometric series $\sum c_{v} e^{i v x}$. This sum appearing in formula (7) converges everywhere, since any $x$ belongs to $K_{j}$ for all sufficiently large $j$ and for such $j$

$$
\left|\psi\left(m_{j}\right) e^{i m_{j} x} Q_{j}(x)\right| \leq\left|\psi\left(m_{j}\right)\right| \cdot\left|\frac{1}{2^{j} \psi\left(m_{j}\right)}\right|=\frac{1}{2^{j}} .
$$

Since the terms of $S$ have distinct frequencies and since the constant term of $Q_{j}(x)$ is 1 , we have

$$
c_{m_{j}}=\psi\left(m_{j}\right) .
$$

The proof of Theorem 6 is complete. 
We now pass to the consideration of particular subsequences of partial sums. The underlying phenomenon that makes the two dimensional example happen is the fact that for any fixed $x$ with $|x|<\pi$, as $n \rightarrow \infty, \sin ^{2 n}(x / 2)$ decays exponentially, even though the constant term of its (finite) Fourier series, $\frac{1}{4^{n}}\left(\begin{array}{c}2 n \\ n\end{array}\right) \simeq \frac{1}{\sqrt{\pi n}}$, decays very slowly. We thank Benjamin Muckenhoupt for suggesting that we also look at this phenomenon in one dimension.

We will consider the particular subsequences $S_{2^{j}}$ and $S_{\left[j^{p}\right]}$ for $1<p<\infty$.

Theorem 7. If the subsequence $\left\{S_{2^{j}}\right\}$ of a trigonometric series converges almost everywhere, then the coefficients must grow more slowly than exponentially. This result is best possible in the sense that if we are given a sequence $\varphi(n)$ growing more slowly than exponentially, there is a trigonometric series which has the subsequence of partial sums $\left\{S_{2^{j}}\right\}$ converging everywhere, but whose coefficients grow like $\varphi(n)$.

Proof. We will need the following lemma.

Lemma 8. Let $\left\{P_{\alpha}(x)\right\}$ be a set of trigonometric polynomials. Suppose that for each $\lambda \in(0,1)$, there is a positive constant $B=B(\lambda)$ such that for each $\alpha,\left|P_{\alpha}(x)\right| \leq$ $B$ for all $x$ in some subset of $(-\pi, \pi]$ of measure at least $2 \pi \lambda$. Then for each $\gamma>1$, there is a constant $b=b(\gamma)>0$, such that for every $\alpha$ and every $x$, $\left|P_{\alpha}(x)\right| \leq b \gamma^{\operatorname{degree}\left(P_{\alpha}\right)}[\mathrm{AW}, 404-406]$.

Let $S$ be a trigonometric series with the property that $\left\{S_{2^{k}}\right\}$ converges almost everywhere. At almost every $x$ we have $S(x)=\lim _{n \rightarrow \infty} S_{2^{n}}(x)$, where

$$
\begin{gathered}
S_{2^{n}}(x)=c_{0}+c_{1} e^{i x}+c_{-1} e^{-i x}+\sum_{j=1}^{n} A_{j}(x), \\
A_{j}(x)=P_{j}(x) e^{i\left(2^{j-1}+1\right) x}+Q_{j}(-x) e^{-i\left(2^{j-1}+1\right) x},
\end{gathered}
$$

and $P_{j}$ and $Q_{j}$ are trigonometric polynomials of degree $2^{j-1}-1$. For each $j$, let $B_{j}(x)=e^{i 2^{j} x} A_{j}(x)$ and note that $B_{j}$ is a trigonometric polynomial of degree $2^{j+1}$. Since $S_{2^{n}}$ converges almost everywhere, the sequence $\left\{B_{j}(x)\right\}$ converges to 0 almost everywhere. By Egoroff's theorem, given any $\lambda \in(0,1)$, there is a set $E \subset[-\pi, \pi),|E|>2 \pi \lambda$, such that the sequence $\left\{B_{j}\right\}$ converges to 0 uniformly on $E$. Since each $B_{j}$ is a trigonometric polynomial and consequently a bounded function, it follows that there is a constant $B=B(E)$ such that $\left|B_{j}(x)\right| \leq B$ for all $j$ and for all $x \in E$. By Lemma 8 , for each $\gamma>1$ there is a $b=b(\gamma)$ so that every coefficient $b_{k}^{(j)}$ of $B_{j}$ satisfies

$$
\left|b_{k}^{(j)}\right|=\left|\frac{1}{2 \pi} \int B_{j}(x) e^{-i k x} d x\right| \leq\left\|B_{j}\right\|_{\infty} \leq b \gamma^{2^{j+1}} .
$$

Now think of $S$ as a trigonometric series of the form $\sum c_{k} e^{i k x}$. Whenever $|k| \in$ $\left[2^{j-1}+1,2^{j}\right], c_{k}$ is a coefficient of $B_{j}$, so that

$$
\left|c_{k}\right| \leq b \gamma^{2^{j+1}}=b\left\{\left(\gamma^{4}\right)^{|k|}\right\}^{\frac{2^{j-1}}{|k|}} \leq b\left(\gamma^{4}\right)^{|k|},
$$

whence $\limsup _{k \rightarrow \infty}\left|c_{k}\right|^{1 /|k|} \leq \gamma^{4}$. Letting $\gamma \rightarrow 1$ shows that the coefficients of $S$ grow more slowly than exponentially. 
Conversely, let $\varphi(n)$ be any sequence growing more slowly than exponentially. The required counterexample is

$$
S(x)=\sum_{j=2}^{\infty} A_{j}(x),
$$

where $A_{j}(x)=\varphi\left(3 \cdot 2^{j-2}\right) P_{j}(x) e^{i\left(2^{j-1}+1\right) x}, P_{j}(x)=\sqrt{\pi} \ell^{3 / 2} \sin ^{2 \ell}\left(\frac{x}{2}\right)\left(1+e^{i x}\right) e^{i \ell x}$, and $\ell=2^{j-2}-1$. Stirling's formula shows that the $\ell$-th coefficient of the $2 \ell$-th degree trigonometric polynomial $P_{j}$,

$$
\sqrt{\pi} \ell^{3 / 2} \frac{1}{(2 i)^{2 \ell}}\left\{\left(\begin{array}{c}
2 \ell \\
\ell
\end{array}\right)-\left(\begin{array}{c}
2 \ell \\
\ell-1
\end{array}\right)\right\}
$$

is asymptotic to 1 as $j \rightarrow \infty$. But the $\ell$-th coefficient of $P_{j}$, when multiplied by $\varphi\left(3 \cdot 2^{j-2}\right)$, is exactly the $3 \cdot 2^{j-2}$-th coefficient of $S$ thought of as a trigonometric series. In other words, if we write $S=\sum c_{k} e^{i k x}$, then $c_{3 \cdot 2^{j-2}} \simeq \varphi\left(3 \cdot 2^{j-2}\right)$ as $j \rightarrow \infty$.

It only remains to show that $S$, as given in equation (8), converges everywhere on $[-\pi, \pi)$. This is clear when $x=-\pi$, since $P_{j}(-\pi)=0$ for all $j$. If $|x|<\pi$, then $a=\left|\sin \left(\frac{x}{2}\right)\right|$ is less than 1 . Thus,

$$
\begin{aligned}
\left|A_{j}(x)\right|^{\frac{1}{2^{j}}} & \leq\left(\varphi\left(3 \cdot 2^{j-2}\right) \sqrt{\pi} \ell^{3 / 2} a^{2 \ell}(1+1)\right)^{\frac{1}{2^{j}}} \\
& =(2 \sqrt{\pi})^{\frac{1}{2^{j}}}\left(2^{j-2}-1\right)^{\frac{3}{2^{j-2}-1} \cdot \frac{2^{j-2}-1}{2^{j+1}}} \varphi\left(3 \cdot 2^{j-2}\right)^{\frac{1}{3 \cdot 2^{j-2}} \cdot \frac{3 \cdot 2^{j-2}}{2^{j}}} a^{\frac{2^{j-1}-2}{2^{j}}} .
\end{aligned}
$$

Since $\frac{2^{j-2}-1}{2^{j+1}} \leq 1, \frac{3 \cdot 2^{j-2}}{2^{j}} \leq 1$, and $\varphi$ is slowly varying, it follows that $\underset{j \rightarrow \infty}{\limsup }\left|A_{j}(x)\right|^{\frac{1}{2^{j}}}$ $\leq a$, so that $\sum A_{j}(x)$ converges by comparison with the convergent series $\sum a^{2^{j}}$.

In order to discuss some other specific sequences we now introduce a scale of sequences describing various growth rates which are less than exponential. For each $q>0$, define $G_{q}$ to be the set of sequences $\left\{\psi_{n}\right\}$ satisfying $\underset{n \rightarrow \infty}{\limsup }\left|\psi_{n}\right|^{\frac{1}{n^{q}}} \leq 1$. For example,

$$
e^{\frac{n}{\log n}} \in G_{1} \backslash \bigcup_{0<q<1} G_{q}, \quad e^{\frac{n^{1 / 2}}{\log n}} \in G_{1 / 2} \backslash \bigcup_{0<q<1 / 2} G_{q}, \quad \text { and } \quad n^{100} \in \bigcap_{0<q \leq 1} G_{q} .
$$

We find it useful to think of $G_{q}$ as the sequences that "grow more slowly than $e^{n^{q}}$." In particular, $G_{1}$ is exactly the class of sequences growing more slowly than exponentially.

Theorem 9. Let $p$ satisfy $1<p<\infty$. If the subsequence $\left\{S_{\left[n^{p}\right]}\right\}$ of a trigonometric series of power series type converges almost everywhere, then the coefficients of the series must grow more slowly than some function in $G_{\frac{p-1}{p}}$. This result is best possible in the sense that if we are given any sequence $\psi \stackrel{p}{(n)}$ in $G_{\frac{p-1}{p}}$, there is a trigonometric series of power series type which has the subsequence of partial sums $\left\{S_{\left[n^{p}\right]}\right\}$ converging everywhere, but whose coefficients grow as badly as $\psi(n)$.

Remarks. In the statement of this theorem, the brackets around $n^{p}$ denote the greatest integer function.

Since $\lim _{p \rightarrow \infty} \frac{p-1}{p}=1$, Theorem 7 can be thought of as a limiting case $(p=$ $\infty)$ of this result. Similarly, since $\lim _{p \rightarrow 1} \frac{p-1}{p}=0$, the ordinary Cantor-Lebesgue theorem can be thought of as the other limiting case $(p=1)$ if we define $G_{0}$ to be 
the class of all sequences tending to zero. With this definition we have, however, that $\bigcap_{0<q \leq 1} G_{q}$ properly contains $G_{0}$.

There are similar theorems regarding convergence on sets of positive, but not full, measure. For example, if we replace $\varphi\left(3 \cdot 2^{j-2}\right)$ by $2^{\left(3 \cdot 2^{j-2}\right)}$ in the example of Theorem 7 , then the subsequence $\left\{S_{2^{j}}\right\}$ converges whenever $\left|\sin \left(\frac{x}{2}\right)\right|<\frac{1}{2}$, i.e., on an open interval of measure $\frac{2 \pi}{3}$, but has coefficients which grow like $2^{n}$. This is best possible in a sense similar to that of the last two theorems. (Compare the remark on page 410 of $[\mathrm{AW}]$.) We will not dwell on this point.

Proof. Fix $p>1$. We may write $S=c_{0}+\sum_{j=1}^{\infty} P_{j}(x) \exp \left(i\left(\left[(j-1)^{p}\right]+1\right) x\right)$, where for each $j$, the degree of the trigonometric polynomial $P_{j}$ is $\left[j^{p}\right]-\left[(j-1)^{p}\right]-1$.

Let any $\gamma>1$ be given. Uniformizing and applying Lemma 8 in a manner similar to the proof of Theorem 7 , we find that if we think of $S$ as a trigonometric series $\sum c_{k} e^{i k x}$, then there is a constant $b(\gamma)$ such that whenever $k \in\left[\left[(j-1)^{p}\right]+1,\left[j^{p}\right]\right]$,

$$
\left|c_{k}\right| \leq b \gamma^{\left[j^{p}\right]-\left[(j-1)^{p}\right]-1} .
$$

Using the mean value theorem and the fact that $p>1$, we have

$$
\left[j^{p}\right]-\left[(j-1)^{p}\right]-1 \leq j^{p}-(j-1)^{p}=p(j-\theta)^{p-1}<p j^{p-1} .
$$

So, letting $\ell=(1 / k)^{(p-1) / p}$,

$$
\left|c_{k}\right|^{\ell} \leq b^{\ell} \gamma^{p j^{p-1} \ell}
$$

But the exponent of $\gamma$ is less than or equal to $p\left(\frac{j}{j-1}\right)^{p-1}$, which tends to $p$ as $j$ tends to $\infty$, so that $\underset{j \rightarrow \infty}{\limsup }\left|c_{k}\right|^{\ell} \leq \gamma^{p}$. Finally, let $\gamma \rightarrow 1$ to get $\underset{j \rightarrow \infty}{\limsup }\left|c_{k}\right|^{1 / k^{(p-1) / p}}$ $\leq 1$.

Conversely, let $\psi \in G_{\frac{p-1}{p}}$ and consider the series

$$
S(x):=\sum_{j=1}^{\infty} \psi\left(\left[j^{p}\right]+1+k\right) P_{j}(x) \exp \left(i\left(\left[j^{p}\right]+1\right) x\right),
$$

where $k=k(j)$ is the largest integer such that $\left[j^{p}\right]+1+2 k+1 \leq\left[(j+1)^{p}\right]$ and $P_{j}(x)=$ $\sqrt{\pi}(k)^{3 / 2} \sin ^{2 k}\left(\frac{x}{2}\right) e^{i k x}\left(1+e^{i x}\right)$. Notice that $P_{j}$ is a trigonometric polynomial of degree $2 k+1$ and its $k$-th coefficient, $d_{k}=\sqrt{\pi}(k)^{3 / 2} \frac{1}{2^{2 k}}\left(\left(\begin{array}{c}2 k \\ k\end{array}\right)-\left(\begin{array}{c}2 k \\ k-1\end{array}\right)\right)$, is asymptotic to 1 as $j$ tends to $\infty$. Then the $J$-th partial sum of the series $S$, as written above, coincides with the $\left[(J+1)^{p}\right]$-th partial sum of $S$ written in the form $\sum_{n=1}^{\infty} c_{n} e^{i n x}$. As in the proof of Theorem 7 , we again have $S(-\pi)=0$. If $|x|<\pi$ and $a=\left|\sin \left(\frac{x}{2}\right)\right|$, then

$$
\begin{aligned}
& \underset{j \rightarrow \infty}{\limsup }\left|\psi\left(\left[j^{p}\right]+1+k\right) P_{j}(x) e^{\left(i\left(\left[j^{p}\right]+1\right) x\right)}\right|^{\frac{1}{2 k}} \\
& \quad \leq \limsup _{j \rightarrow \infty}\left|\psi\left(\left[j^{p}\right]+1+k\right)\right|^{\frac{1}{2 k}} \cdot a \\
& \quad \leq \limsup _{j \rightarrow \infty}\left|\psi\left(\left[j^{p}\right]+1+k\right)\right|^{\frac{1}{\left(\left[j^{p}\right]+1+k\right)^{\frac{p-1}{p}}} \frac{\left(\left[j^{p}\right]+1+k\right)^{\frac{p-1}{p}}}{2 k}} \cdot a .
\end{aligned}
$$


But this is $\leq a$ since $\psi \in G_{\frac{p-1}{p}}$ and $2 k \simeq p j^{p-1}$ implies that $\frac{\left(\left[j^{p}\right]+1+k\right)^{\frac{p-1}{p}}}{2 k} \simeq \frac{1}{p}$. Thus $S$ converges provided $\sum a^{C j^{p-1}}$ converges for $C>0$. Since $p>1$, this follows from the integral test. Since $S$ converges everywhere, the subsequence of $\left[J^{p}\right]$-th partial sums of the corresponding trigonometric series do also. Nevertheless,

$$
c_{\left[j^{p}\right]+1+k}=\psi\left(\left[j^{p}\right]+1+k\right) d_{k} \simeq \psi\left(\left[j^{p}\right]+1+k\right) .
$$

We finish by using the above technique to demonstrate the breakdown of the one dimensional Cantor-Lebesgue theorem for subsequences with unbounded gaps. A similar example also appears as example 5 of $[\mathrm{AKR}]$.

Theorem 10. Let $\left\{m_{j}\right\}$ be an increasing sequence of integers with $\sup \left\{m_{j+1}-m_{j}\right\}$ $=\infty$. Then there is a trigonometric series whose $m_{j}$-th partial sums converge everywhere, but whose coefficients do not tend to zero.

Proof. The by now familiar proof consists of sliding powers of $\sin \left(\frac{x}{2}\right)$ into the gaps. Explicitly, choose a sequence $\left\{j_{v}\right\}$ in such a way that $m_{j_{v}+1}-m_{j_{v}}$ is strictly increasing. Let $\ell_{v}$ be the largest integer satisfying $m_{j_{v}}+1+2 \ell_{v}+1 \leq m_{j_{v}+1}$, and form the function

$$
\sum_{v=1}^{\infty} \ell_{v}^{2} \sin ^{2 \ell_{v}}\left(\frac{x}{2}\right) e^{i \ell_{v} x}\left(1+e^{i x}\right) e^{i\left(m_{j_{v}}+1\right) x} .
$$

Just as in many of the above examples, any $m_{J}$-th partial sum of the associated trigonometric series is equal to a partial sum of this series as written. The series as written converges everywhere, but the $\left(m_{j_{v}}+1+\ell_{v}\right)$ th coefficient of the series thought of as a trigonometric series is asymptotic to a constant multiple of $\sqrt{\ell_{v}}$.

Remark. Notice that the three counterexamples appearing in Theorems 7, 9, and 10 all use powers of $\sin \left(\frac{x}{2}\right)$, all are of power series type, and all converge uniformly on $[-\pi+\delta, \pi-\delta]$ for each positive $\delta$.

\section{REFERENCES}

[AW] J. M. Ash and G. V. Welland, Convergence, uniqueness, and summability of multiple trigonometric series, Trans. Amer. Math. Soc. 163(1972), 401-436. MR 45:9057.

[AFR] J. M. Ash, C. Freiling, and D. Rinne, Uniqueness of rectangularly convergent trigonometric series, Ann. of Math. 137(1993), 145-166. MR 93m:42002

[AKR] J. M. Ash, R. P. Kaufman, and E. Rieders, The Cantor-Lebesgue property, Israel J. Math. 84(1993), 179-191. MR 94m:42007

[B] J. Bourgain, Spherical summation and uniqueness of multiple trigonometric series, Internat. Math. Res. Notices 1996, no. 3, 93-107. CMP 96:11

[C1] P. J. Cohen, Topics in the theory of uniqueness of trigonometrical series, Thesis, University of Chicago, Chicago, IL, 1958.

[C2] Bernard Connes, Sur les coefficients des séries trigonométriques convergentes sphériquement, C. R. Acad. Sci. Paris, Sér. A, 283(1976), 159-161. MR 54:10975.

[C3] R. Cooke, A Cantor-Lebesgue theorem in two dimensions, Proc. Amer. Math. Soc., 30(1971), 547-550. MR 43:7847.

[R] W. Rudin, Real and Complex Analysis, 3rd ed., McGraw-Hill, New York, 1987. MR 88k:00002

[S] V. L. Shapiro, Uniqueness of multiple trigonometric series, Ann. of Math. 66(1957), 467480. MR 19:854; MR 19:1432.

Department of Mathematics, DePaul University, Chicago, Illinois 60614-3504

E-mail address: mash@math.depaul.edu

E-mail address: gwang@math.depaul.edu 